\title{
Pilot of an in vivo fluoride-releasing device
}

\author{
In vitro and in vivo assessment of a glass slow fluoride releasing device: a pilot study
}

\section{E. J. Curzon and K. J. Toumba Br Dent J 2004; 196: 543-546}

\section{Aims}

The aims were to evaluate a) whether a slow release fluoride-glass pellet (SFG) would be retained in the mouth and release fluoride (F) over a long period of time, $\mathrm{b}$ ) what concentrations of $\mathrm{F}$ in the glass would provide ideal intra-oral saliva $F$ concentrations and c) whether an SFG would affect blood plasma concentrations of $\mathrm{F}$ after swallowing compared with ingestion of a commercial $\mathrm{NaF}$ tablet.

\section{Methods}

a) A prototype SFG was attached to a maxillary molar of a volunteer. Baseline saliva F concentrations were measured prior to glass placement, daily in week one; one day a week for weeks two to three and then one day a month up to 18 months. Four subjects had the SFG for six months with saliva F concentration assessments at periodic intervals. b) SFGs containing $F$ at 13.3\%, 18.3\% and $21.9 \%$, and an improved solubility, were tested using three volunteers and saliva F concentrations measured. c) Five volunteers each swallowed either a SFG or a NaF tablet. Blood plasma samples were taken at baseline and $\mathrm{F}$ measured at time intervals of $2.5,5,10,20,30,45,60,90$ and 120 mins post-ingestion.

\section{Results}

a) The prototype SFG were successfully retained and released F into saliva; mean concentrations of $0.035 \mathrm{mg} \mathrm{L}^{-1}$ were achieved lasting for over 18 months. Overall saliva F concentrations were approximately doubled. Analysis of the pellet at the end of use showed it still contained some F possibly indicating a recharging effect. b) The 13.3\% F concentration SFG produced significantly higher saliva $\mathrm{F}$ levels than the two other concentrations tested. The two higher concentration $\mathrm{F}$ glasses contained aluminium as part of the formulation of the glass structure, which is known to bind $\mathrm{F}$ whereas the $13.3 \%$ glass contained $\mathrm{F}$ alone. c) While blood plasma $\mathrm{F}$ levels increased after ingestion of the NaF tablet there was no increase in $\mathrm{F}$ when the SFG was swallowed.

\section{Conclusion}

A slow release $\mathrm{F}$ containing glass device showed promise as a means to enhance intra-oral F saliva concentration.

\section{IN BRIEF}

- New technology for caries prevention.

- Targeted fluoride for at-risk children.

- Long-term caries prevention delivery system.

\section{COMMENT}

It has been a long time since there was a real breakthrough in fluoride therapy. Following the introduction of water fluoridation in the United States some 60 years ago, fluoride has been used as a caries preventive agent through oral ingestion and topical applications either by healthcare workers or by individuals. While the efficacy of water fluoridation has been proven, communities still resist the introduction of this measure. Also, while professional application of fluoride or school rinsing programmes work, they are time-consuming, costly and often not affordable by health authorities. Self-application of fluoride relies on compliance and certainly in poor communities may not be affordable. So the time is ripe for the introduction of an effective, cheap, long-lasting method to provide fluoride in the oral cavity.

This paper describes just such a method and, in my opinion, is an important advance in preventive dentistry. Like so many good ideas it is simple. The paper presents evidence to show that the device produced an increase in salivary fluoride over 18 months. This pilot study followed a classical and ethical approach. The primary author first tried the device on himself and when this showed promise, five volunteers increased the study sample. There is clear evidence presented to show that caries preventive levels of fluoride are produced in saliva without raising plasma fluoride levels.

The authors believe that the slow release device has use in high risk individuals. I agree with this. However, living and working in a developing country as I do, I foresee a wider role in oral public health. It is conceivable that attachment of the slow release device to teeth besides being done by dentists or oral hygienists could be done by trained staff such as school or clinic nurses or even members of a community.

An unexpected spin-off from the device was reported recently, at the 2003 ORCA meeting, of a strong effect on reducing dentine hypersensitivity. ${ }^{1}$

The researchers are to be congratulated on their innovative work. I look forward to the results of trials in high caries risk individuals after which I have no doubt the device will be adopted worldwide.

P. Cleaton-Jones, Professor of Experimental Odontology and Director, South African Medical Research Council / University of the Witwatersrand Dental Research Institute, Johannesburg

1. Toumba KJ, Andreadis G A. Alleviation of dentine sensitivity using slow release fluoride devices: a pilot study. Caries Res 2003; 37: 273, ORCA abstract 19.

doi:10.1038/sj.bdj.4811239 